\title{
Modeling of Thermo-Mechanical Behavior of Saturated Clays
}

\author{
E.L. Liu $^{1,2}$ and H.L. Xing ${ }^{2}$ \\ ${ }^{1}$ School of Mechanics \& Civil Engineering, China University of Mining \& Technology, \\ Beijing, P.R. China \\ lelo2@mails.tsinghua.edu.cn \\ ${ }^{2}$ Quakes, University of Queensland, St.Lucia, Brisbane, QLD 4072, Australia
}

\begin{abstract}
A new thermo-elasto-plastic constitutive model of saturated clay is proposed here based on the double hardening model for clays and available experimental results, which describes the effects of temperature and over consolidation ratio on the mechanical properties of saturated clays by introducing the two hardening parameters $-\sigma_{c}^{\prime} \& \alpha$. Finally, the proposed model is applied to model the related important features of saturated clays under the different temperatures and loadings and compared with the available experimental results to demonstrate its accuracy.
\end{abstract}

Keywords: Thermo-mechanical behavior, Saturated clay, Constitutive model.

\section{Introduction}

Recently more attention has been focused on researching the mechanical response of soils to the combined effects of stress and temperature which is important in many geotechnical engineering applications. A number of experiments were carried out to investigate the influence of temperature on the behaviors of soils ${ }^{[1][2]}$. To study the effects of temperature on clays, a few of constitutive models were proposed to describe the thermo-elasto-plastic behavior of the solid skeleton ${ }^{[3][4]}$. Most of the above models mainly focused on modeling the thermal-mechanical behaviors of saturated soils under the constant loading, but without considering the different overconsolidated ratio (OCR) effects under the isothermal triaxial drained conditions. In this paper, we propose a new thermo-elasto-plastic constitutive model to describe the combined effects of the temperature and the OCR on the mechanical properties of saturated clays and verify it with the available experimental results.

\section{Thermo-Mechanical Constitutive Equations of Saturated Clay}

\subsection{The Formulation of the Constitutive Equations}

Based on the above experimental results, a thermo-elastic-plastic constitutive equation is deduced in the following. Let 


$$
\sigma_{m}^{\prime}=\frac{1}{3} \sigma_{k k}^{\prime} ; \sigma_{s}=\sqrt{\frac{3}{2} s_{i j} s_{i j}}, s_{i j}=\sigma_{i j}^{\prime}-\sigma_{k k}^{\prime} \delta_{i j} ; \varepsilon_{v}=\varepsilon_{k k} ; \varepsilon_{s}=\sqrt{\frac{2}{3} e_{i j} e_{i j}}, e_{i j}=\varepsilon_{i j}-\frac{1}{3} \varepsilon_{k k} \delta_{i j}
$$

The thermo-elastic strain tensor $\varepsilon^{T e}$ is the superposition of a reversible thermal induced strain $\varepsilon^{T}$ and a mechanical elastic strain $\varepsilon^{e}$ under adiabatic conditions. In incremental form we have

$$
d \varepsilon^{T e}=d \varepsilon^{T}+d \varepsilon^{e}
$$

The elastic shear strain is assumed as purely mechanical, so we have

$$
d \varepsilon_{v}^{T e}=\frac{d \sigma_{m}^{\prime}}{K}+\beta_{s}^{\prime} d T \quad \text { and } \quad d \varepsilon_{s}^{e}=\frac{d \sigma_{s}}{3 G}
$$

where $d \varepsilon_{v}^{T e}$ is the volumetric thermo-elastic strain increment, $d \varepsilon_{s}^{e}$ is the shear strain increment, $d \sigma_{m}^{\prime}$ is the mean effective stress increment, $d \sigma_{s}$ is the shear stress increment, $d T$ is the temperature increment, $\beta_{s}^{\prime}$ is the isotropic thermal expansion coefficient of the solid skeleton, $K$ is bulk elastic modulus and $G$ is shear elastic modulus.

The thermal-plastic strain depends on deformation and temperature history, and in general cannot be uniquely expressed through effective stress and temperature. Based on the double hardening model for clays ${ }^{[5]}$ under the isothermal temperature condition, the irreversible thermal effects are introduced using the dependence of yield surface, flow rule and internal variables on temperature. The yield surface is assumed to be a function of stress, plastic volumetric strain and plastic shear strain and temperature variation $d T$ (referring to an environmental temperature $T_{o}$ ),

$$
F=\frac{\sigma_{m}^{\prime}}{1-\left(\frac{\eta}{\alpha}\right)^{n}}-\sigma_{c}^{\prime}=0
$$

where $\eta=\sigma_{s} / \sigma_{m}^{\prime} ; \sigma_{c}^{\prime}$ and $\alpha$ are hardening parameters,

$$
\begin{gathered}
\sigma_{c}^{\prime}=\sigma_{c}^{\prime}(T) \exp \left(\beta \varepsilon_{v}^{p}\right)=\sigma_{c}^{\prime}\left(T_{o}\right) \exp \left(\beta \varepsilon_{v}^{p}\right)\left\{1-\gamma \log \left[T / T_{o}\right]\right\} \\
\alpha=\alpha\left(\varepsilon_{s}^{p}, T\right)=\alpha_{c}(T)\left[\alpha_{m}-\left(\alpha_{m}-\alpha_{o}\right) \exp \left(\frac{\varepsilon_{s}^{p}}{c_{a}}\right)\right]
\end{gathered}
$$

In which

$$
\varepsilon_{v}^{p}=\varepsilon_{k k}^{p}, \varepsilon_{s}^{p}=\sqrt{\frac{2}{3} e_{i j}^{p} e_{i j}^{p}}, e_{i j}^{p}=\varepsilon_{i j}^{p}-\frac{1}{3} \varepsilon_{k k}^{p} \delta_{i j} ;
$$

$\sigma_{c}^{\prime}(T)$ and $\sigma_{c}^{\prime}\left(T_{o}\right)$ are the pre-consolidation stress at a given temperature $T$ and $T_{o}$, respectively; $\alpha_{c}(T)$ represents the effect of temperature on the hardening parameter $\alpha$; $\gamma, \beta, \alpha_{m}, \alpha_{o}$ and $c_{a}$ are constant for a certain soil.

The incremental thermal-plastic strain can be formulated as the following equations 


$$
d \varepsilon_{i j}^{p}=d \lambda \frac{\partial g\left(\sigma_{i j}^{\prime}, \varepsilon_{i j}^{p}, \Delta T\right)}{\partial \sigma_{i j}^{\prime}} \text { or } d \varepsilon_{v}^{p}=d \lambda \frac{\partial g}{\partial \sigma_{m}^{\prime}} d \varepsilon_{s}^{p}=\frac{3}{2} d \lambda \frac{\partial g}{\partial \sigma_{s}}
$$

where $g$ is a plastic potential,

$$
g=g\left(\sigma_{m}^{\prime}, \sigma_{s}, \varepsilon_{v}^{p}, \varepsilon_{s}^{p}, \Delta T\right)
$$

And $d \lambda$ is the plastic multiplier, which can be derived from the consistency conditions,

$$
\frac{\partial F}{\partial \sigma_{i j}^{\prime}} d \sigma_{i j}^{\prime}+\frac{\partial F}{\partial \varepsilon_{v}^{p}} d \varepsilon_{v}^{p}+\frac{\partial F}{\partial \varepsilon_{s}^{p}} d \varepsilon_{s}^{p}+\frac{\partial F}{\partial T} d T=0
$$

Substituting for the plastic volumetric strain increment $d \varepsilon_{v}^{p}$ and the plastic shear strain $d \varepsilon_{s}^{p}$ from the equations (7), $d \lambda$ can be described as,

$$
d \lambda=\frac{\frac{\partial F}{\partial \sigma_{i j}^{\prime}} d \sigma_{i j}^{\prime}+\left[\frac{\partial F}{\partial \sigma_{c}^{\prime}} \frac{\partial \sigma_{c}^{\prime}}{\partial T}+\frac{\partial F}{\partial \alpha} \frac{\partial \alpha}{\partial T}\right] d T}{H}
$$

where $H$ is thermo-plastic hardening modulus, and

$$
H=\frac{3}{2} \frac{n \sigma_{m}^{\prime} \eta^{n}}{\alpha^{n+1}\left[1-\left(\frac{\eta}{\alpha}\right)^{n}\right]^{2}} \frac{1}{c_{a}}\left[\alpha-\alpha_{c}(T) \alpha_{m}\right] \frac{\partial g}{\partial \sigma_{s}}+\beta \sigma_{c}^{\prime} \frac{\partial g}{\partial \sigma_{m}^{\prime}}
$$

The total volume strain increment $d \varepsilon_{v}$ and shear strain increment $d \varepsilon_{s}$ are assumed as,

$$
d \varepsilon_{v}=d \varepsilon_{v}^{T e}+d \varepsilon_{v}^{p}, d \varepsilon_{s}=d \varepsilon_{s}^{e}+d \varepsilon_{s}^{p}
$$

Combining equations (7), (10), (11) with (12), the incremental form of stressstrain-temperature equations can be described finally as,

$$
d \sigma_{m}^{\prime}=C_{m v} d \varepsilon_{v}+C_{m s} d \varepsilon_{s}+B_{T v} d T, d \sigma_{s}=C_{s v} d \varepsilon_{v}+C_{s s} d \varepsilon_{s}+B_{T s} d T
$$

Where $C_{m v}=\frac{1}{M}\left[\frac{1}{3 G}+\frac{3}{2} \frac{1}{H} \frac{\partial F}{\partial \sigma_{s}} \frac{\partial g}{\partial \sigma_{s}}\right], C_{m s}=-\frac{1}{M} \frac{1}{H} \frac{\partial F}{\partial \sigma_{s}} \frac{\partial g}{\partial \sigma_{m}^{\prime}}, C_{s v}=-\frac{3}{2} \frac{1}{M} \frac{1}{H} \frac{\partial F}{\partial \sigma_{m}^{\prime}} \frac{\partial g}{\partial \sigma_{s}}$,

$$
\begin{aligned}
C_{s s}= & \frac{1}{M}\left[\frac{1}{K}+\frac{1}{H} \frac{\partial F}{\partial \sigma_{m}^{\prime}} \frac{\partial g}{\partial \sigma_{m}^{\prime}}\right], M=\frac{1}{3 K G}+\frac{1}{3 G H} \frac{\partial F}{\partial \sigma_{m}^{\prime}} \frac{\partial g}{\partial \sigma_{m}^{\prime}}+\frac{3}{2 K H} \frac{\partial F}{\partial \sigma_{s}} \frac{\partial g}{\partial \sigma_{s}}, \\
B_{T v}= & -\frac{1}{M}\left[\frac{1}{3 G} \beta_{s}^{\prime}+\frac{3}{2} \frac{1}{H} \frac{\partial F}{\partial \sigma_{s}} \frac{\partial g}{\partial \sigma_{s}} \beta_{s}^{\prime}+\frac{1}{3 G H}\left(\frac{\partial F}{\partial \sigma_{c}^{\prime}} \frac{\partial \sigma_{c}^{\prime}}{\partial T}+\frac{1}{H} \frac{\partial F}{\partial \alpha} \frac{\partial \alpha}{\partial T}\right) \frac{\partial g}{\partial \sigma_{m}^{\prime}}\right] \\
+ & \frac{1}{M}\left[\frac{3}{2} \frac{1}{H^{2}}\left(1-\frac{1}{H}\right) \frac{\partial F}{\partial \sigma_{s}} \frac{\partial F}{\partial \alpha} \frac{\partial \alpha}{\partial T} \frac{\partial g}{\partial \sigma_{m}^{\prime}} \frac{\partial g}{\partial \sigma_{s}}\right], \\
B_{T s}= & -\frac{1}{M}\left[\frac{3}{2 H K}\left(\frac{\partial F}{\partial \sigma_{c}^{\prime}} \frac{\partial \sigma_{c}^{\prime}}{\partial T}+\frac{\partial F}{\partial \alpha} \frac{\partial \alpha}{\partial T}\right) \frac{\partial g}{\partial \sigma_{s}}+\frac{3}{2} \frac{1}{H^{2}}\left(1-\frac{1}{H}\right) \frac{\partial F}{\partial \sigma_{m}^{\prime}} \frac{\partial F}{\partial \alpha} \frac{\partial \alpha}{\partial T} \frac{\partial g}{\partial \sigma_{m}^{\prime}} \frac{\partial g}{\partial \sigma_{s}}\right] \\
& +\frac{1}{M}\left[\frac{3}{2} \frac{1}{H} \frac{\partial F}{\partial \sigma_{m}^{\prime}} \frac{\partial g}{\partial \sigma_{s}} \beta_{s}^{\prime}\right] .
\end{aligned}
$$




\subsection{The Determination of the Model Parameters}

For the thermo-elastic case, the three sets of parameters $K, G$ and $\beta_{s}^{\prime}$, can be determined in this paper as follows,

$$
K=K_{r e f}\left(\frac{\sigma_{m}^{\prime}}{P_{a}}\right)^{m} G=G_{r e f}\left(\frac{\sigma_{m}^{\prime}}{P_{a}}\right)^{m}
$$

where $K_{r e f}$ and $G_{r e f}$ are the bulk and shear modulus at a reference pressure; $P_{a}=0.104 \mathrm{MPa}$, and $m=a_{1}\left(\sigma_{c} / \sigma_{o}\right)+b_{1}, a_{1}, b_{1}$ are constants, $\sigma_{c}$ is consolidated pressure, $\sigma_{o}$ is unit pressure and OCR is the over-consolidation ratio.

$$
\beta_{s}^{\prime}=\beta_{s o}^{\prime}(3.5-0.2(O C R))(1+\varsigma T)(O C R)
$$

In which $\beta_{s o}^{\prime}$ is the volumetric thermal expansion coefficient at the reference temperature, and $\varsigma$ corresponds to the slope of the variation of $\beta_{s}^{\prime}$ with respect to present temperature $T$.

While for the thermo-plastic parameters, due to the lack of experimental results, the thermo-plastic potential function is assumed to be the same as the yield function

$$
g=F \text { and } \alpha_{c}(T)=\alpha_{c}\left(T_{o}\right)+N_{s}\left(T-T_{o}\right)
$$

Where $\alpha_{c}\left(T_{o}\right)$ is the reference value at the reference temperature $T_{o}, N_{s}$ is a constant. Other parameters in equation (7) can be determined as follows: $\alpha_{m}=\sqrt[n]{1+n} \sin \varphi_{r}$ and $\varphi_{r}$ is residual frictional angle; $n=a_{2}\left(\sigma_{c} / \sigma_{o}\right)+b_{2}, a_{2}, b_{2}, \alpha_{o}$ and $c_{a}$ are constants.

\section{Validation of the Proposed Model}

The validation tests were conducted on CM clay (Kaolin) under triaxial shear conditions with different temperatures ${ }^{[6]}$. These comparisons were made at two different temperatures for two over-consolidation ratios $(\mathrm{OCR}=1.2$ and 3). Figure 1 and Figure 2 show the results for the isothermal triaxial cases conducted at $22{ }^{\circ} \mathrm{C}$ and $90{ }^{\circ} \mathrm{C}$, respectively. The model parameters used are: $\sigma_{c}^{\prime}\left(T_{o}\right)=0.6 \mathrm{MPa}$, $\beta_{s o}^{\prime}=-1.3 \times 10^{-5}\left({ }^{\circ} C^{-1}\right), \varsigma=-0.003, \beta=5.2, \gamma=0.17, K_{r e f}=1.65 \mathrm{MPa}, G_{r e f}=0.92 \mathrm{MPa}$, $C_{a}=0.5, \varphi_{r}=23^{\circ}, \alpha_{o}=\alpha_{m}, \alpha_{c}\left(T_{o}\right)=2.52, N_{s}=-0.03, a_{1}=1.33, b_{1}=0.835$, $a_{2}=2.33, b_{2}=0.035$. All the above results demonstrated that the proposed model can reproduce the major aspects of the temperature influence on mechanical behavior of saturated clays and agrees well with the experimental results. 

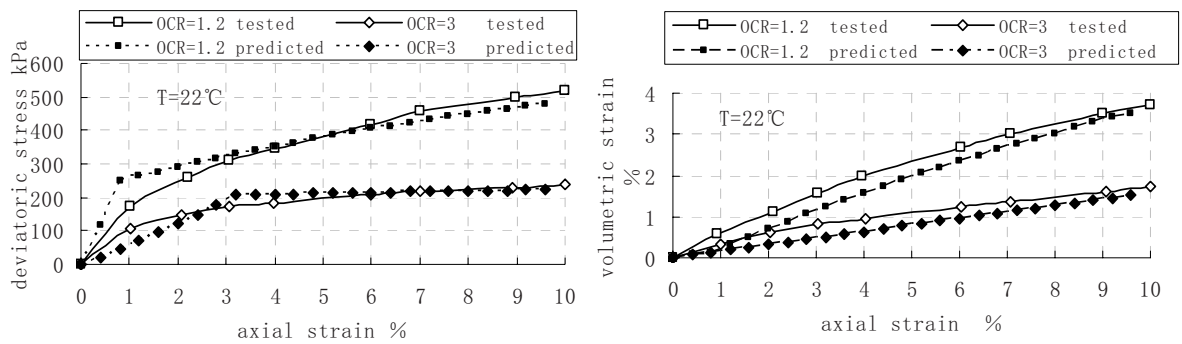

Fig. 1. Comparison results on drained triaxial case at $22^{\circ} \mathrm{C}$ for two $\mathrm{OCR}$ values
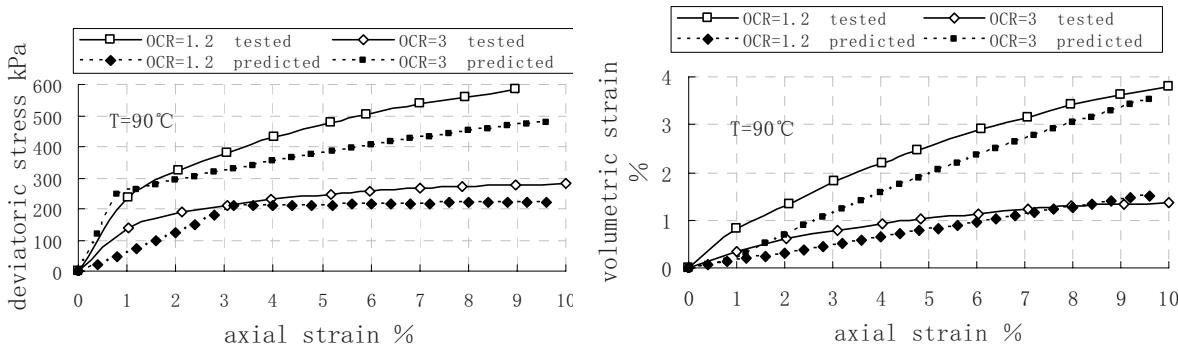

Fig. 2. Comparison results on drained triaxial case at $90^{\circ} \mathrm{C}$ for two $\mathrm{OCR}$ values

\section{Conclusions}

The effects of both the temperature and the over consolidation ratio (OCR) on the mechanical properties of saturated clays are described by introducing two hardening parameters. The proposed model has been applied to compare with the experimental results, which demonstrated that the new model may describe many important thermoelastic-plastic features of saturated clays well.

\section{References}

1. Baldi, G., Theckel, T., Pellegrini, R.: Thermal Volume Changes of The Mineral-water System in Low-porosity Clay Soils. Can. Geo. J. 25 (1988) 807-825

2. Sultant, N.,Delage,P.,Cui,Y.J.: Temperature Effects on The Volume Change Behavior of Boom Clay. Engineering Geology 64 (2002) 135-145

3. Hueckel, T., Borsetto, M.: Thermo-plasticity of Saturated Soils and Shales : Constitutive Equations. J. of Geo. Engineering 12 (1990) 1765-1777

4. Cui, Y.J.,Sultan, N., Delage, P.: A Thermo-mechanical Model for Saturated Clays. Can. Geo. J. 37 (2000) 607-620

5. Shen, Z.J.: A Double Hardening Model for Clays. Rock and Soil Mechanics 1 (1995)1-8 (in Chinese)

6. Cekerevac, C., Laloui, L.: Experimental Study of Thermal Effects on The Mechanical Behavior of A Clay. Int.J. for Num. and Anal. Met. in Geo. 28 (2004) 209-228 